\title{
氮杂环卡宾配位的双核钴碳基配合物催化炔炋与三级硅烷的 马氏选择性硅氢化反应
}

\author{
陈杰坪陆 展* \\ (浙江大学化学系 杭州 310027)
}

\section{Markovnikov Hydrosilylation of Alkynes with Tertiary Silanes via $\mathrm{N}$-Heterocyclic Carbene-Promoted Dinuclear Cobalt Carbonyl Catalysis}

\author{
Chen, Jieping Lu, Zhan* \\ (Department of Chemistry, Zhejiang University, Hangzhou 310027)
}

烯基硅化合物是一类重要的原料, 被广泛用于有机 合成、高分子化学和材料科学领域 ${ }^{[1]}$. 过渡金属催化炔 烃的直接硅氢化反应是合成烯基硅化合物最高效和最 原子经济性的方法之一. 因此, 该反应受到越来越广泛 的关注 ${ }^{[2]}$. 然而, 炔烃与硅烷化合物的硅氢化反应过程 中存在着化学选择性和区域选择性问题(图 1). 例如炔 烃可以和硅烷反应生成半氢化的副产物, 炔烃可以发生 单次硅氢化生成烯基硅产物, 也可能发生过度转化生成 双硅化合物. 同时, 炔烃的硅氢化可以生成三种不同的 加成产物, 即马氏加成产物 ( $\alpha$-烯基硅)和反马氏加成产 物( $\beta$ - $(E)$-烯基硅和 $\beta$-(Z)-烯基硅). 因此, 实现炔烃硅氢 化的选择性调控是一个具有挑战性的难题.

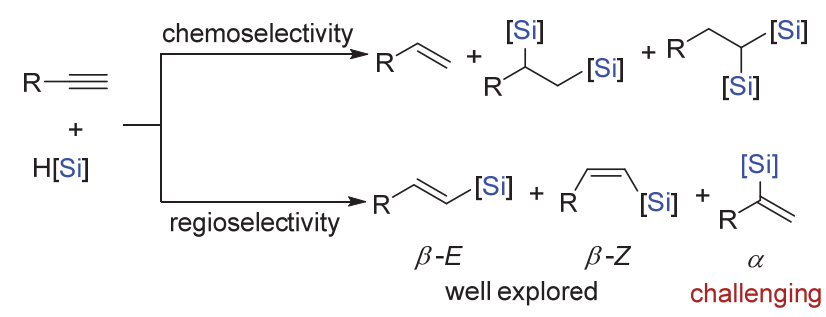

图 1 炔烃的硅氢化反应

Figure 1 Hydrosilylation of alkynes

在以往文献报道中, 金属催化炔烃的硅氢化反应一 般得到反马氏选择性的产物. 目前, 金属催化炔烃的马 氏选择性硅氢化反应报道例子较少. 2001 年, Trost 课题 组 ${ }^{[3]}$ 报道了 $\left[\mathrm{CpRu}(\mathrm{MeCN})_{3}\right]\left[\mathrm{PF}_{6}\right]$ 催化的非导向末端炔烃
的硅氢化反应. 随后, 陆展 ${ }^{[4]}$ 和黄正课题组 ${ }^{[5]}$ 各自独立 报道了地球丰产金属钴催化剂催化炔烃与一级或二级 硅烷的硅氢化反应，高效制备 $\alpha$-烯基硅化合物，但这些 催化剂一般不适用于三级硅烷. Pawluc 和 Isobe 课题组 ${ }^{[5]}$ 分别报道了利用三级硅烷的炔烃马氏选择性硅氢化反 应，但这些反应存在底物范围受限以及反应选择性差等 问题. 因此, 发展地球丰产金属催化剂催化炔烃与三级 硅烷的区域选择性硅氢化反应具有重要意义.

最近, 中国科学院上海有机化学研究所邓亮团队 ${ }^{[6]}$ 利用羰基双钴氮杂环卡宾配合物 $\left[(\mathrm{IPr})_{2} \mathrm{Co}_{2}(\mathrm{CO})_{6}\right]$ 作为预 催化剂, 实现了末端炔烃与三级硅烷的马氏选择性硅氢 化反应(图 2). 该催化体系可以用于高效制备 $\alpha$-烯基硅 和含硅聚合物, 并具有反应选择性高、官能团兼容性好 以及底物范围广等优点. 机理研究表明, 单氮杂环卡宾 (NHC)-二钴物种可能是反应过程中真正的催化活性物 种; 催化体系中炔烃独特的配位模式以及 NHC 配体的 空间位阻特性导致反应具有较高的 $\alpha$-选择性.

作者对该催化体系的底物适用范围进行探索时发 现，反应具有很好的底物兼容性. 带有醇、醚、卤代烃、 胺、腈、酯等取代基的芳香炔烃、烷基炔烃均能参与反 应, 并具有较高的选择性. 同时, 反应体系也可以兼容 多种三级硅烷. 但对于大位阻的炔烃, $\beta-(E)$ 的产物比例 显著提升. 利用这种方法, 作者进行了克级产物的合成, 并且实现了其后续的转化, 通过 Hiyama 偶联制备了高 附加值的 1,1-二取代烯烃化合物.

* Corresponding author. E-mail: luzhan@zju.edu.cn. Published online August 23, 2021. 


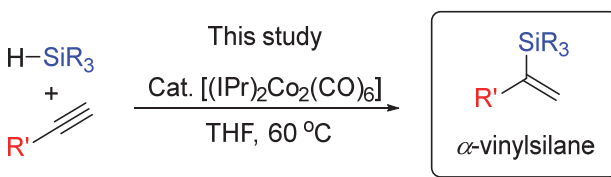

- $\alpha$-selective $\bullet$ high yields $\cdot \mathrm{R}^{\prime}=$ alkyl, aryl groups

- $\mathrm{HSiR}_{3}=\mathrm{HSiEt}_{3}, \mathrm{HSi}(\mathrm{OEt})_{3}, \mathrm{HSiPh}_{3}, \mathrm{HSiMe}_{2} \mathrm{Cl}$ $\mathrm{HSi}\left(\mathrm{OSiMe}_{3}\right)_{2} \mathrm{Me}, \mathrm{HMe}_{2} \mathrm{SiOSiMe}_{2} \mathrm{H}$

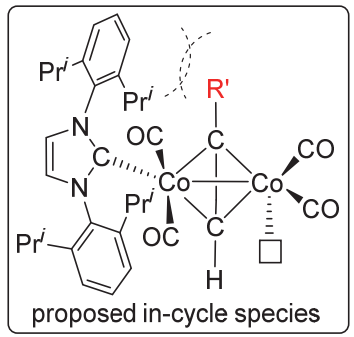

图 $2\left[(\mathrm{IPr})_{2} \mathrm{Co}_{2}(\mathrm{CO})_{6}\right]$ 催化的炔烃硅氢化反应

Figure 2 Hydrosilylation of alkynes catalyzed by [(IPr) $2-$ $\left.\mathrm{Co}_{2}(\mathrm{CO})_{6}\right]$

作者进一步对反应的机理进行探究. 预催化剂 1 的 化学计量反应, 钴配合物 $1 \sim 5$ 的催化反应动力学实验 和氛代实验表明, 单(NHC)-二钴物种可能是反应过程
中真正的催化物种. 预催化剂 1 与苯乙炔在 $60{ }^{\circ} \mathrm{C}$ 下反 应，可以分离得到炔桥二钴配合物 2 和 3.1 与三乙基硅 烷在 $60{ }^{\circ} \mathrm{C}$ 下反应，可以分离得到钴硅配合物 4. 单 (NHC)二钴炔桥配合物 2 可以与硅烷在室温下反应生成 $\alpha$-烯基硅以及钴硅配合物 4. 配合物 2 与硅烷反应生成 钴硅物种 4 , 而不是 $(\mathrm{IPr}) \mathrm{Co}_{2}(\mathrm{CO})_{5}$, 表明高活性的(IPr)$\mathrm{Co}_{2}(\mathrm{CO})_{5}$ 一旦形成, 可能会迅速与硅烷反应生成配合物

4. 相比于配合物 2 , 配合物 3 需要在 $60{ }^{\circ} \mathrm{C}$ 下才能与硅 烷反应，生成 $\alpha$-烯基硅及二(NHC)-四羰基二钴配合物 5(图 3). 动力学实验表明，单(NHC)-二钴炔桥配合物 2 与苯乙炔、硅烷的催化活性高于其他配合物 $1,3 \sim 5$. 氛 代实验表明反应经历了 $\mathrm{Si}-\mathrm{H}$ 的顺式加成过程.

根据相应的实验结果，作者提出了可能的反应机 理. 在诱导期内, 预催化剂 $\mathbf{1}$ 与炔烃反应产生 $\mathbf{2}$ 和 $\mathbf{3}$. 配 合物 2 和 3 中的 CO、IPr 配体解离生成中间体 6 , 硅烷 与 6 配位生成 7 , 然后经历 $\sigma$-复分解生成炔桥钴硅物种, 然后烯烃解离形成中间体 8,8 与炔烃反应再生成活性中 间体 6. 由于氮杂环卡宾配体和 $\mathrm{R}^{\prime}$ 取代基的空间位阻, 使得在配合物 9 中, $\angle 1\left(144.6^{\circ}\right)$ 远大于 $\angle 2\left(124.9^{\circ}\right)$. 在

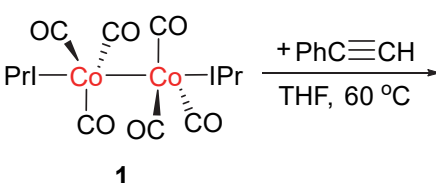

$+2 \mathrm{HSiEt}_{3} \downarrow \mathrm{THF}, 60^{\circ} \mathrm{C}$<smiles>CCOC(C=O)(C=O)C(=O)c1ccccc1</smiles>

4, isolated yield: $50 \%$

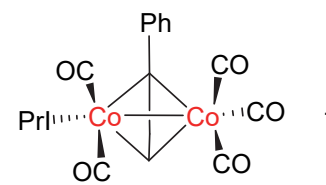

2 , isolated yield: $8 \%$

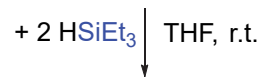

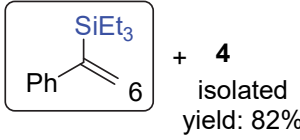

NMR yield: $93 \%$

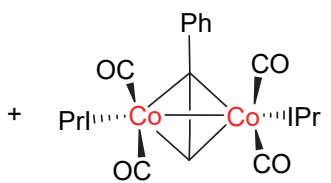

3, isolated yield: $10 \%$ $+2 \mathrm{HSiEt}_{3} \mid \mathrm{THF}, 60^{\circ} \mathrm{C}$

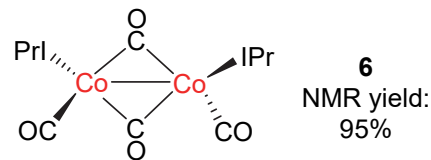

5, isolated yield: $73 \%$

图 3 钴催化硅氢化反应的机理研究

Figure 3 Mechanistic studies on the cobalt-catalyzed hydrosilylation reaction
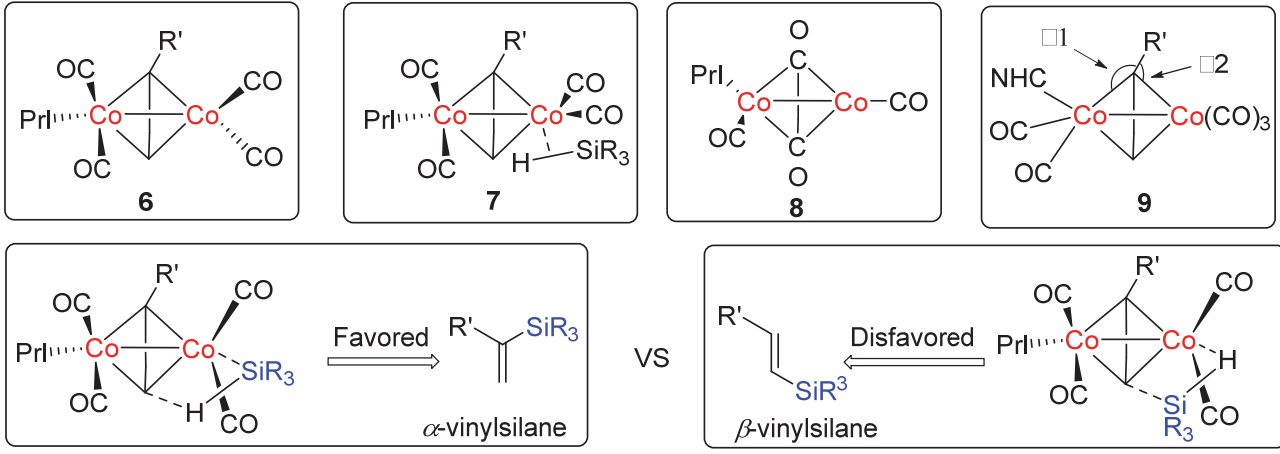

图 4 硅烷与钴-碳键可能的作用模式

Figure 4 Possible interaction modes of hydrosilane with $\mathrm{Co}-\mathrm{C}$ bonds 
这种空间环境下, $\mathrm{HC}-\mathrm{Co}(\mathrm{CO})_{2}$ 与 $\mathrm{H}-\mathrm{SiR}_{3}$ 发生的 $\sigma$-复分 解反应具有一定的立体选择性(图 4). 由于硅取代基与 炔烃取代基的空间互斥, 在四元环过渡态中, 硅取代基 倾向于位于钴的 $\alpha$ 位而非 $\beta$ 位. 因此, 这种空间位阻使 得反应的选择性以马氏选择性为主. 同时, 这也解释了 使用位阻较小的氮杂环卡宾配体(IMes)会使反应区域选 择性变差; 使用大位阻的炔烃底物, 会使反应倾向于生 成反马氏硅氢化的产物.

该结果不仅为合成全取代烯基硅提供了一种有效 的方法, 而且还提出了硅氢化反应的新机制, 为后续的 选择性反应提供了参考依据.

\section{References}

[1] Almeida, L. D.; Wang, H.; Junge, K.; Cui, X.; Beller, M. Angew. Chem., Int. Ed. 2021, 60, 550.

[2] (a) Sun, J.; Deng, L. ACS Catal. 2016, 6, 290.

(b) Chen, J.; Guo, J.; Lu, Z. Chin. J. Chem. 2018, 36, 1075. (c) Cheng, Z.; Guo, J.; Lu, Z. Chem. Commun. 2020, 56, 2229.

[3] Trost, B. M.; Ball, Z. T. J. Am. Chem. Soc. 2001, 123, 12726.

[4] Guo, J.; Lu, Z. Angew. Chem., Int. Ed. 2016, 55, 10835.

[5] Zuo, Z.; Yang, J.; Huang, Z. Angew. Chem., Int. Ed. 2016, 55, 10839 .

[6] (a) Skrodzki, M.; Patroniak, V.; Pawluc, P. Org. Lett. 2021, 23, 663. (b) Huang, K.-H.; Isobe, M. Eur. J. Org. Chem. 2014, 2014, 4733.

[7] Wang, D.; Lai, Y.; Wang, P.; Leng, X.; Xiao. J, Deng, L. J. Am. Chem. Soc. 2021, 143, 12847. 